Influence de la salinité et de la concentration initiale sur le tassement d'une vase de Loire

Younes Ouraq

Chercheur, LMG, Université de Nantes

Martines Sanchez

Maître de conférence, LMG, Université de Nantes

Alain Grovel

Professeur des universités, LMG, Université de Nantes

Résumé

L'objectif de ce travail est de déterminer les facteurs qui peuvent influencer le processus de tassement des vases dans les milieux estuariens. L'influence de la salinité et de la concentration initiale est étudiée en laboratoire. Les essais sont interprétés par une méthode modifiée de Kynch qui permet de relier le coefficient de perméabilité à la concentration de la vase grâce à la mesure de la vitesse de tassement en surface. Lors des essais on observe un ralentissement important de la vitesse de sédimentation si les vases ne sont pas saturées, lequel s'explique par une poussée verticale ascendante due à la présence d'une phase gazeuse dans la vase.

\title{
Introduction
}

Sous l'action de la pesanteur les flocons en suspension dans l'eau décantent. Arrivés au fond ils s'écrasent sous leur propre poids. Progressivement une partie de l'eau de leur structure va s'évacuer. Le réseau se resserre et forme une suspension dont la concentration moyenne va s'accroitre avec le temps. Pendant le tassement la structure interne des sédiments va subir plusieurs changements allant d'une structure lâche (nid d'abeille) vers une structure compacte et dense.

L'évolution de la concentration moyenne du culot de tassement en fonction du temps (Fig.1) permet de distinguer plusieurs phases successives (Migniot 1968) . $\mathrm{Au}$ début les particules élémentaires constituant la vase vont s'agglomérer pour former des agrégats dont la vitesse de chute est nettement plus importante que celle des particules à l'état individuel c'est la floculation. Ensuite, on observe successivement : la chute des flocons à vitesse entravée, la première phase de tassement avec l'élimination d'une partie de l'eau interstitielle non liée aux particules sédimentaires par des forces électriques, la deuxième phase de tassement plus lente, correspondant à l'évacuation de l'eau par des puits de drainage et enfin, la troisième phase de tassement très lente, qui se traduit par un arrangement de la structure du dépôt et une perte d'eau des couches adsorbées.

D'après Migniot (1968) la concentration moyenne varie selon une fonction logarithmique du temps, dont les coefficients dépendent de la phase du tassement, du complexe vase-eau ainsi que du milieu aqueux. 
Dans cette étude les essais de tassement en colonnes réalisés en laboratoire, sont analysés à partir des courbes expérimentales de tassement par une méthode de Kynch modifiée (Kynch, 1952; Tan et al, 1990). L'origine des temps de la courbe de tassement est corrigé afin de tenir compte de la phase de floculation (Sanchez et Grovel, 1994).

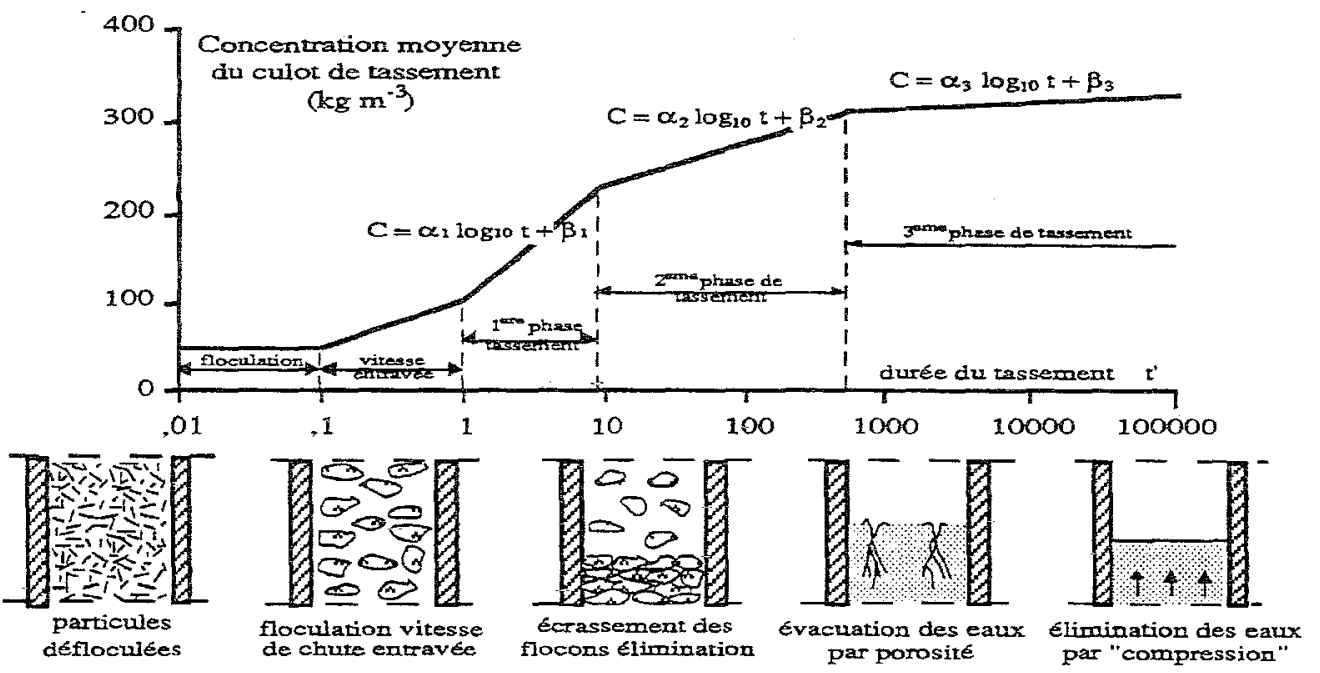

Fig. 1.- Graphique montrant les différentes phases du tassement (d'après Migniot, 1989).

\section{Essais de tassement}

On reproduit en laboratoire les conditions de tassement de la vase en procédant comme suit : dans un premier temps un échantillon de vase fraîche issue du port de plaisance de Trentemoult en Loire est débarrassé de la fraction grossière à l'aide d'un tamis de $1 \mathrm{~mm}$, ensuite cette vase est soumise à une agitation afin de l'homogénéiser.

L'étude de l'évolution au cours du temps des niveaux des culots de tassement ayant des concentrations initiales comprises entre 50 et $200 \mathrm{~g} / 1$ pour des salinités variant entre $0 \mathrm{~g} / \mathrm{kg}$ et $30 \mathrm{~g} / \mathrm{kg}$ permet de reproduire dans des tubes transparents de diamètre $9 \mathrm{~cm}$ un grand nombre de cas rencontrés dans la nature. L'ensemble des essais est réalisé à partir d'une hauteur initiale de $100 \mathrm{~cm}$ à une température constante de $21^{\circ} \mathrm{C}$. Chaque série de mesures comporte cinq échantillons ayant la même concentration initiale et cinq salinités différentes. Nous avons constaté dans la première série d'essais de tassement ayant pour concentration initiale $\mathrm{Ci}=200 \mathrm{~g} / \mathrm{l}$ et pour les différentes salinité $(\mathrm{S}=0 ; 5 ; 10 ; 20 ; 30 \mathrm{~g} / \mathrm{kg}$ ) une surélévation au cours du temps du niveau de l'eau claire par rapport à la hauteur initiale du complexe vaseeau (Fig.3). Cette augmentation du volume total de la mixture montre bien la présence d'une phase gazeuse dans la vase sous forme d'air mélangé à de la vapeur d'eau et du gaz carbonique ; ce qui explique le ralentissement important du tassement qui a été observé pour cette série de mesures. Pour ces essais la vase ne peut plus être considérée comme un complexe saturé.

Lors de nos essais la mesure du niveau de la couche de vase est effectuée régulièrement à des intervalles de temps croissants allant jusqu'à onze jours. Ceci 
permet par la suite d'ajuster un modèle simulant le tassement et de quantifier linfluence de la salinité initiale et de la concentration initiale sur ce processus.

\section{Tassement de vases saturées}

\subsection{Infuence de la concentration initiale sur le tassement}

La figure 2, représente l'évolution en fonction du temps de l'épaisseur des dépôts de vase ayant des concentrations initiales de $175 ; 150 ; 100$ et $50 \mathrm{~g} / 1$, une hauteur initiale de $1 \mathrm{~m}$ et une salinité de $30 \mathrm{~g} / \mathrm{kg}$. Les mêmes courbes ont été construites pour des salinités de $0 ; 5 ; 10$ et $20 \mathrm{~g} / \mathrm{kg}$.

En général, on fait les observations suivantes :

-la vitesse de tassement est au début d'autant plus grande que la concentration initiale est plus faible.

-les courbes de tassement des différentes mixtures montrent une tendance vers une asymptote d'autant plus rapidement que la concentration initiale est faible. Ces mêmes observations ont été émises par d'autres auteurs.

Salinité : $30 \mathrm{~g} / \mathrm{kg}$

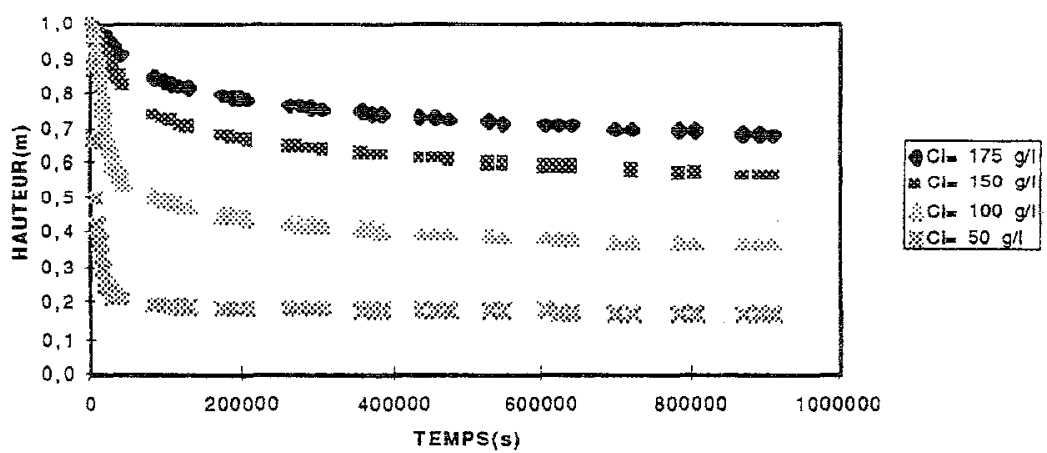

Fig. 2.- Courbes de tassement pour une salinité constante de $30 \mathrm{~g} / \mathrm{kg}$ et pour des concentrations initiales variant entre $50 \mathrm{~g} / \mathrm{l}$ et $175 \mathrm{~g} / \mathrm{l}$.

\subsection{Influence de la salinité sur le tassement.}

Dans cette partie on étudie le tassement à concentration initiale constante et à salinité variable $(0 ; 5 ; 10 ; 20$ et $30 \mathrm{~g} / \mathrm{kg})$. La figure.3, montre les courbes de tassement correspondant à une concentration initiale de $175 \mathrm{~g} / 1$. Les principales observations sont les suivantes :

-le tassement est d'autant plus rapide au début du processus que la salinité est plus importante. Ce phénomène serait lié à une floculation plus intense en présence du sel; de même la tendance vers l'asymptote est accélérée avec l'augmentation de la salinité.

-l'influence de la salinité est très nette pour les mixtures de concentration initiale 50 $\mathrm{g} / \mathrm{l}$; elle diminue progressivement avec l'augmentation de la concentration initiale. Ainsi la concentration moyenne du culot passe de 143 g/l à $236 \mathrm{~g} / \mathrm{l}$ après dix heures 
de tassement lorsque la salinité varie de $0 \mathrm{~g} / \mathrm{kg}$ à $30 \mathrm{~g} / \mathrm{kg}$ pour une mixture de concentration initiale $50 \mathrm{~g} / \mathrm{l}$ alors qu'elle ne varie que très peu pour une concentration initiale de $175 \mathrm{~g} /$.

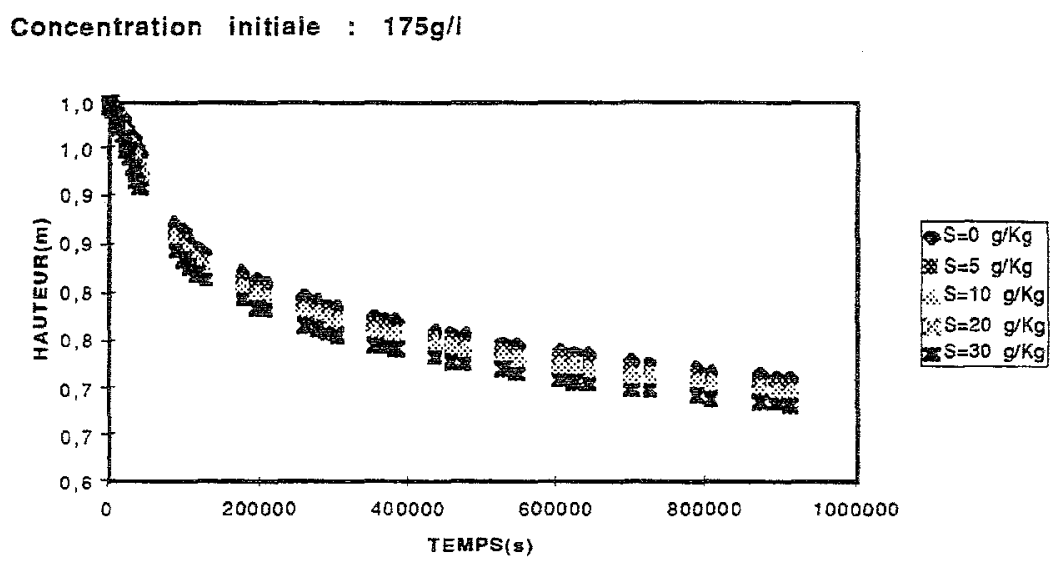

Fig. 3.- Courbes de tassement pour une concentration initiale de $175 \mathrm{~g} /$, une hauteur initiale de $1 \mathrm{~m}$ et des salinités de $0 ; 5 ; 10 ; 20$ et $30 \mathrm{~g} / \mathrm{kg}$.

\subsection{Temps caractéristique de la floculation}

Au début du processus de tassement, la vitesse de sédimentation de la vase augmente graduellement vers la vitesse finale associée à la concentration initiale Afin de tenir compte de ce phénomène lié à la floculation, on corrige l'origine de l'axe des temps et l'on évalue un facteur temps To caractéristique de la floculation (voir Fig.4) .

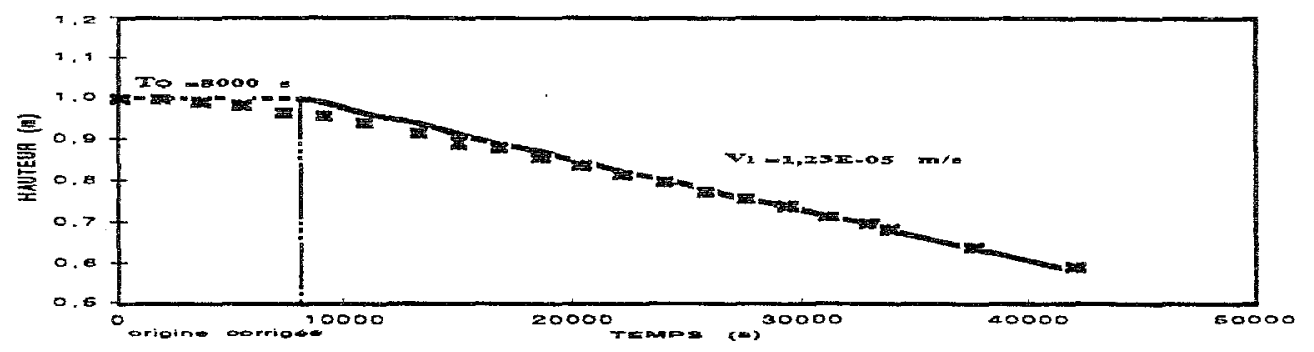

Fig. 4.- Détermination graphique du temps caractéristique de la floculation $(\mathrm{Ci}=100 \mathrm{~g} / \mathrm{l} ; \mathrm{S}=0 \mathrm{~g} / \mathrm{kg})$

\begin{tabular}{|c|c|c|c|c|}
\hline$\overline{\mathrm{S}}$ & $50 \mathrm{~g} / 1$ & $100 \mathrm{~g} / \mathrm{l}$ & $150 \mathrm{~g} / \mathrm{I}$ & $175 \mathrm{~g} /$ \\
\hline $0 \mathrm{~g} / \mathrm{kg}$ & $\overline{0 s}$ & $8000 \mathrm{~s}$ & $4510 \mathrm{~s}$ & $4150 \mathrm{~s}$ \\
\hline $5 \mathrm{~g} / \mathrm{kg}$ & $0 \mathrm{~s}$ & $4300 \mathrm{~s}$ & $2680 \mathrm{~s}$ & $1320 \mathrm{~s}$ \\
\hline $10 \mathrm{~g} / \mathrm{kg}$ & $0 \mathrm{~s}$ & $3380 \mathrm{~s}$ & $2060 \mathrm{~s}$ & $600 \mathrm{~s}$ \\
\hline $20 \mathrm{~g} / \mathrm{kg}$ & $0 \mathrm{~s}$ & $3080 \mathrm{~s}$ & $1460 \mathrm{~s}$ & $0 \mathrm{~s}$ \\
\hline $30 \mathrm{~g} / \mathrm{kg}$ & $\overline{0 \mathrm{~s}}$ & $1850 \mathrm{~s}$ & $1000 \mathrm{~s}$ & $0 \mathrm{~s}$ \\
\hline
\end{tabular}

Tableau. 1.- Temps To caractéristique de la floculation en secondes. 


\subsection{Coefficient de perméabilité des vases saturées}

En admettant que les contraintes effectives restent négligeables pendant le processus de sédimentation, la vitesse de la phase solide est donnée par la relation suivante (Been, 1980):

$$
V_{s}=-\frac{k}{\rho_{f}}\left(1-\frac{\rho_{f}}{\rho_{s}}\right) C
$$

avec:

$\rho_{\mathrm{s}}$ : masse volumique de la phase solide $\left(\mathrm{kg} \mathrm{m}^{-3}\right)$,

$\rho_{f}$ : masse volumique de la phase fluide $\left(\mathrm{kg} \mathrm{m}^{-3}\right)$,

$\mathrm{C}$ : concentration $\left(\mathrm{kg} \mathrm{m}^{-3}\right)$,

$\mathrm{k}$ : coefficient de perméabilité de la vase $\left(\mathrm{m} \mathrm{s}^{-1}\right)$.

On constate que les vases molles suivent souvent une loi de variation en fonction de la concentration qui peut être mise sous la forme suivante (Sanchez, 1992; Tan et al, 1990) :

$$
k=A_{1} \exp \left(-A_{2} \frac{C}{\rho_{s}}\right)
$$

avec:

$A_{1}$ et $A_{2}$ des coefficients propres à chaque matériau $\left(A_{1}>0\right.$ et $\left.A_{2}>0\right)$

La détermination graphique de la vitesse de la phase solide permet d'évaluer l'évolution du coefficient de perméabilité en fonction de la concentration de la vase.

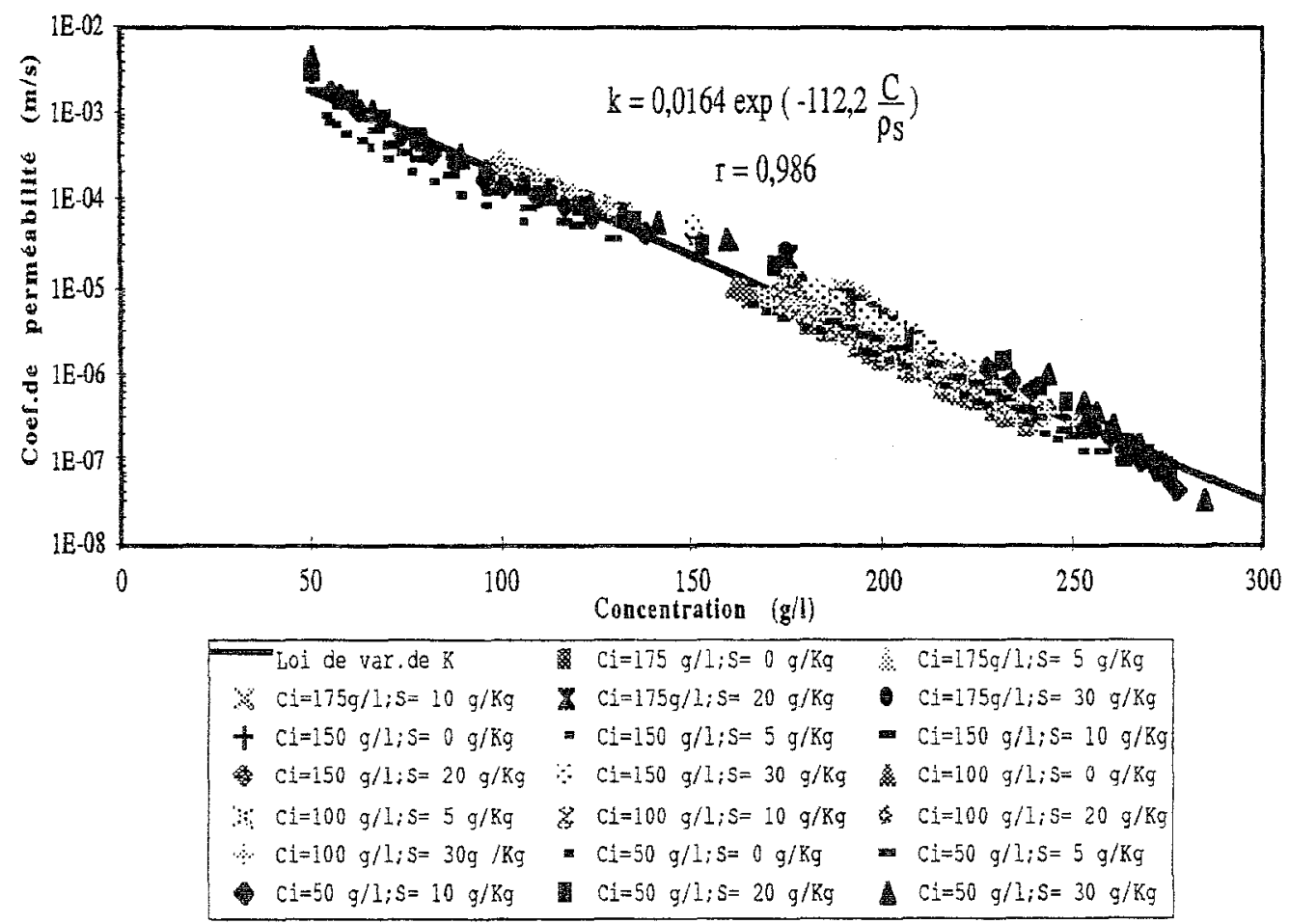

Fig. 5.- loi de variation du coefficient de perméabilité de la vase saturée 
Le lissage des coefficients de perméabilité évalués à partir de la totalité des vingt essais effectués en laboratoire, nous permet de déterminer les coefficients $A_{1}$ et $A_{2}$ de la loi de perméabilité de la vase valable pour un domaine de concentrations variant entre 50 et $300 \mathrm{~g} / \mathrm{l}$; les résultats obtenus sont (Fig.5) :

$A_{1}=0,0164 \mathrm{~m} \mathrm{~s}^{-1}$

$\mathrm{A}_{2}=112,2$

Le coefficient de corrélation ( déterminé par l'erreur entre notre lissage et les vitesses mesurées en laboratoire ) vaut $: r=0,986$

\subsection{Conclusions sur le tassement des vases saturées.}

Mis à part des petits écarts, on constate que l'ensemble des courbes de tassement des vases saturées est modélisé par une seule loi de variation du coefficient de perméabilité en fonction de la concentration, laquelle est propre à cette vase. La différence essentielle réside dans le temps de floculation, variable selon les condition initiales. Ce paramètre $T_{0}$, caractérise le temps que met une suspension de vase pour atteindre sa vitesse limite de sédimentation associée à sa concentration initiale. Les essais montrent en général une diminution du temps de floculation avec l'augmentation de la salinité. Cependant, les floculations plus ou moins rapides selon les cas étudiés conduisent toutes à terme, à la même loi de variation de $\mathrm{k}$ avec la concentration et pratiquement à la même concentration finale lorsque la concentration initiale est fixe.

\section{Vases non saturées}

\subsection{Tassement des vases non saturées}

Les essais de tassement de cette série (Fig.6) ont été réalises sur une vase très fraîche qui contient $10 \%$ de matière organique très active.

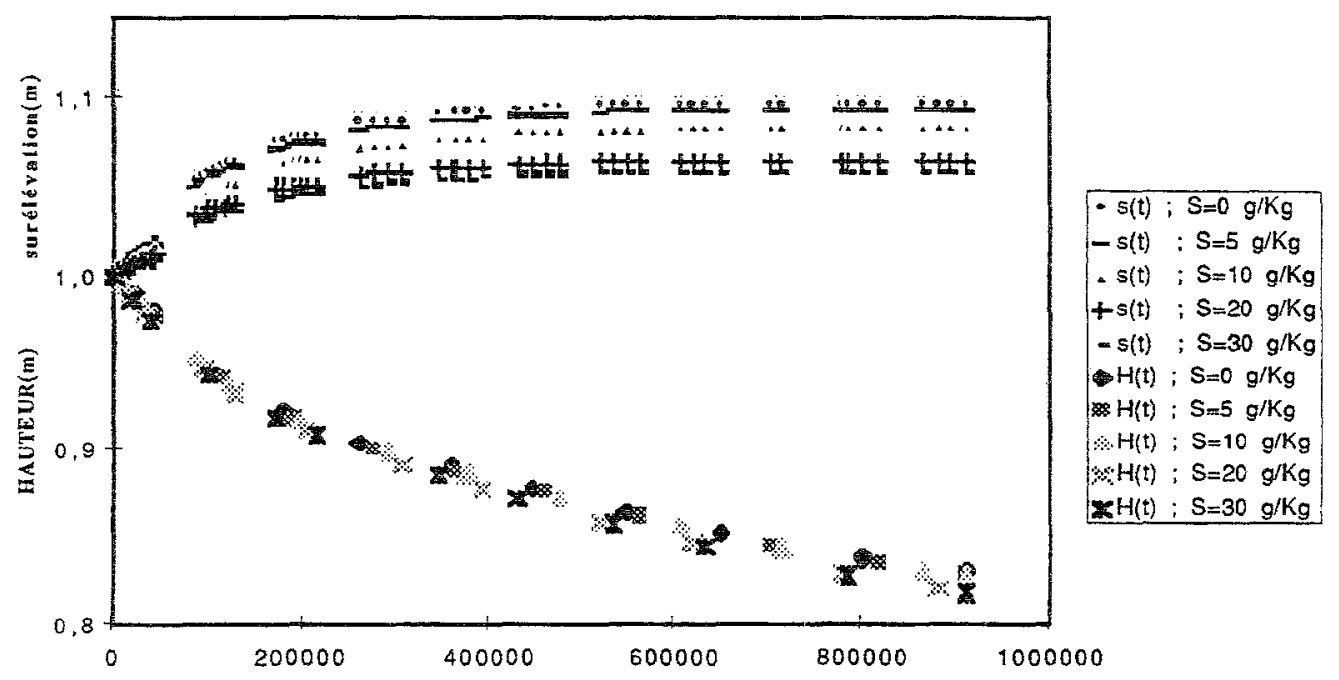

Fig. 6.- Courbes de tassement et surélévation du niveau de l'interface air-eau en fonction du temps pour une vase de Trentemoult (Loire) non saturée ( $\mathrm{Ci}=200 \mathrm{~g} / \mathrm{l}$ ). 
On a constaté un dégagement gazeux résultant de l'action combinée de plusieurs processus biochimiques: activité bactérienne; décomposition de la matière organique ; photosynthèse. Le principal témoignage de la présence d'une phase gazeuse est la surélévation de l'interface eau-air. La présence de la phase gazeuse dans la vase diminue considérablement la vitesse de sédimentation du fait de la poussée verticale ascendante qu'elle provoque. Nous constatons que l'introduction du sel a très peu d'influence sur la vitesse de tassement mais diminue visiblement la quantité de gaz dégagée lors du tassement, aussi l'asymptote de la courbe de tassement est atteinte d'autant plus rapidement que la quantité du sel introduite est plus faible (Fig.6). Ce phénomène peut s'expliquer par une réduction de la décomposition de la matière organique et de l'activité bactérienne par le sel.

\subsection{Vitesse de tassement des vases non saturées}

Afin de tenir compte du volume d'air dans la vase, la relation de Been (1) donnant la vitesse de la phase solide est modifiée de la façon suivante :

$\mathrm{v}_{\mathrm{S}}=-\frac{\mathrm{k}}{\rho_{\mathrm{f}}}\left[\left(1-\frac{\rho_{\text {至 }}}{\rho_{\mathrm{S}}} \frac{\mathrm{S}_{\mathrm{r}}}{100}\right) \mathrm{C}-\left(1-\frac{\mathrm{S}_{\mathrm{r}}}{100}\right) \rho_{\mathrm{f}}\right]$

où $S_{\mathrm{r}}$ est le degré de saturation de la vase en $\%$

La vitesse de tassement deviendrait nulle lorsque l'égalité suivante est satisfaite :

$$
\frac{\left(1-\frac{\rho_{f}}{\rho_{S}} \frac{S_{r}}{100}\right) C}{\left(1-\frac{S_{r}}{100}\right) \rho_{f}}=1
$$

Le rapport entre la vitesse des solides en présence d'une phase gazeuse sur cette vitesse pour une vase saturée est le suivant :

$\frac{v_{S}(S r \neq 100)}{v_{S}(S r=100)}=\frac{\left(1-\frac{\rho_{f}}{\rho_{S}} \frac{S_{r}}{100}\right) C-\left(1-\frac{S_{r}}{100}\right) \rho_{f}}{\left(1-\frac{\rho_{f}}{\rho_{S}}\right) C}$

\begin{tabular}{|c|c|}
\hline & $\frac{\mathrm{v}_{\mathrm{S}}(\mathrm{Sr} \neq 100)}{\mathrm{v}_{\mathrm{S}}(\mathrm{Sr}=100)}$ \\
\hline Salinité & 0,197 \\
\hline $0 \mathrm{~g} / \mathrm{kg}$ & 0,231 \\
\hline $5 \mathrm{~g} / \mathrm{kg}$ & 0,298 \\
\hline $10 \mathrm{~g} / \mathrm{kg}$ & 0,456 \\
\hline $20 \mathrm{~g} / \mathrm{kg}$ & 0,515 \\
\hline $30 \mathrm{~g} / \mathrm{kg}$ & \\
\hline
\end{tabular}

Tabieau. 2.- Paramètre caractérisant le ralentissement de la vitesse de tassement calculé à partir des valeurs moyennes de $S_{\mathrm{r}}$ et $\mathrm{C}$ correspondant à la phase asymptotique du tassement. 


\section{Conclusions}

Les études de tassement effectuées en laboratoire montrent l'importance de la saturation, de la concentration initiale et de la salinité dans le tassement d'une vase de Loire.

Pour les vases saturées l'influence de la concentration initiale sur la vitesse de tassement apparait comme plus grande que celle de la salinité. L'effet de la salinité est plus marquant pendant la phase initiale du tassement et diminue progressivement avec l'augmentation de la concentration initiale. Lorsque l'on examine le phénomène à l'aide d'une théorie de sédimentation de Kynch modifiée, on s'aperçoit que le rôle des conditions initiales est moindre. En effet, l'ensemble de courbes de tassement correspond à une seule loi de variation du coefficient de perméabilité avec la concentration, de même la concentration finale ne varie pas sensiblement avec la salinité lorsque la concentration initiale est constante. L'effet le plus important lié aux conditions initiales est la durée du temps de floculation qui diminue avec l'augmentation de la salinité.

Pour les vases non saturées, La vitesse de tassement n'est que peu influencée par la salinité. Néanmoins, le degré de saturation diminue avec l'augmentation de la salinité. Une intense décomposition de la matière organique ou une activité bactérienne peuvent être à l'origine de la phase gazeuse dans la structure de la vase dont la présence engendre un ralentissement du tassement. Des analyses biochimiques pourrraient permettre de mieux comprendre cette association.

\section{Références bibliographiques.}

1. Been, K. 1980. Stress strain behaviour of a cohesive soil deposited under water. Thesis presented to the University of Oxford, at Oxford, England, in partial fulfillement of the degree of Doctor of Philopsophy.

2. Kynch, G.F. 1952. A theory of sedimentation. Faraday Society Transactions, $48,166-176$.

3. Migniot, C. 1968. Etude des propriétés physiques de differents sédiments très fins et leur comportement sous des des actions hydrodynamiques, La Houille Blanche, No. 7, 1968, pp 591-620.

4. Sanchez, M.1992. Application du modèle iso-concentration de tassement dans la simulation du tassement des vases molles étudiées au Laboratoire Central d'Hydrauliques de France. Rapport de recherche $n^{\circ}$ CFL-92-006,LMGUniversité de Nantes.

5. Sanchez, M. et Grovel, A.1994. Le tassement des vases comme processus sédimentaire. Actes des $3^{\text {èmes }}$ journées nationales de génie civil- génie côtier. Sète-France.

6. Tan, T.S. ,Yong K.Y. ,Leong E.C and Lee S.L 1990. Sedimentation of clayey slurry. Journal of Geotechnical Engineering, 116, 6, 885-898. 